\title{
Sensorization and Intelligent Systems in Energetic Sustainable Environments
}

\author{
Fábio Silva, David Cuevas, Cesar Analide, José Neves and José Marques
}

\begin{abstract}
Sustainability is an important topic of discussion in our world. However, measuring sustainability and assessing behaviors is not always easy. Indeed, and in order to fulfill this goal, in this work it will be proposed a multi-agent based architecture to measure and assess sustainable indicators taken from a given environment. These evaluations will be based on past and present behaviors of the users and the particularities of the setting, leading to the evaluation of workable indicators such as gas emissions, energetic consumption and the users fitting with respect to the milieu. Special attention is given to user interaction and user attributes to calculate sustainable indicators for each type of structure, i.e., the aim of this scheme is to promote sustainability awareness and sustainable actions through the use of sustainable markers calculated in terms of the information gathered from the environment.
\end{abstract}

\section{Introduction}

Ambient Intelligence (AmI) is still considered an emergent technology that may be embedded into environments, making them both sensitive and responsive. In this sense AmI may be used to achieve several objectives inside such environments, e.g., in sustainability assessment, enforcement and suggestion [5]. In fact, there is an increasing source of concern as more researchers make use of computational resources to find sustainable equilibriums. Sustainable models developed in the literature are also focus of research and improvement, although they may differ on their approach. Some of these models use economical metrics in order to assess sustainability, while others follow social and environmental perspectives in a more accurate form [11]. The work presented uses a multi-agent system to obtain information about an environment so that deliberative and reactive decisions concerning

Fábio Silva, David Cuevas, Cesar Analide, José Neves and José Marques Department of Informatics, University of Minho, e-mail: \{f.aandree, davidjfcuevas@gmail.com\}, \{analide; jneves\}@di.uminho.pt, josealbertomarques@gmail.com 
sustainability can be made. Indeed, multi-agent system architectures that foresee these problems, may be found in [9]. Performance tests showed good results for the service discovery in terms of flexibility and interoperability. Decisions are created using reasoning processes upon the data gathered either with machine learning or context-aware computing, so that specialized intelligent decisions may be made for each user present in an AmI setting. With respect to this area of research, Machine Learning inside AmI environments have already been used for feats such as human activity tracking [3]. Current sustainability assessment considers different indicators and sub-models created and used by specialized people. Our work aims to make it simple to assess and determine sustainability indicators in an intelligent environment through the use of multi-agent architecture and environment sensing. It has been demonstrated by previous research that when people are aware of the consequences of their actions in detail, and they are set with an objective, they tend to act in the best manner to attain it, as it was the case with eletrical consumption [4]. It is expected that the use of architectures like the one proposed in this paper causes the same effect on sustainable measures and user behaviour as it was demonstrated for electric consumption and user behaviour.

\section{Previous Study}

In this section there are presented studies related to the research being conducted. As such each category will be presented and a general descritpion will be made.

Sustainability is a subject of concern for the assurance of the steadiness, viability and use of a system. Currently different approaches to measure and assess sustainability were proposed in the literature, with some focusing on an economical perception, while others emphasize on environment or social perspectives [11]. Sustainability indicators have been useful at pointing out unsustainable practices, however, they are not as good to define and guarantee sustainability [7]. A common accepted definition for the notion of sustainability concerns an equilibrium from social, economical and environmental factors. When some of these features cannot be met, the system is not considered sustainable, but it may be pondered viable, bearable or equitable [11]. In contexts like intelligent buildings, there are commitments to build Key Performance Indicators (KPIs) to monitor sustainability, and act as sustainability indicators from information gathered by a panel of experts [2].

Machine Learning (ML) techniques or methods concede the modeling and learning of preferences and habits in different contexts. These methods also sanction the acquiring of past and current trends and predict future results. From information assembled from different environments, ML techniques may derive models of behaviour and interaction based on specialized backgrounds (e.g., users, environment, social interaction or consumption). ML and Data Mining (DM) techniques may also be used to obtain information about user's habits in AmI settings, from data gathered by sensors in the environment, namely using practices such as Sequence Discovery 
[3], Fuzzy Logic [6], Genetic Programming, Multi-Layer Perceptron, Evolutionary Intelligence [8] or combinations of these techniques [12].

Context-Aware Computing is a component of a ubiquitous computing computing environment. One hopes to make personalized decisions based on contextual factors that may lead to different results to the same situation, once placed in different contexts [10]. Evidence of these context-aware systems can be found in e-Commerce, Information Retrieval and Ubiquitous Systems. In these examples context awareness is used to personalize systems and applications to the present user context [1]. Despite the interest in context-aware computing, context definition for applications and systems is still challenging in some situations.

\section{Multi-Agent System for Sustainable Environments}

This case study presented encompasses an intelligent environment and its user interactions in order to obtain information about its current sustainable condition. The source of such interest may be economical challenges or a conscious mind about the future sustainability of his environment, as described in [4]. The user is supposed to do daily routines such as cooking, sleeping or watching television. Nevertheless, the system records each action on the part of the user interactions, and uses such information to reason about existing sustainability indicators and metrics of control concerning the environment i. e., resource utilization, $\mathrm{CO}_{2}$ emissions and of its own attendance on each internal premise in the environment for example.

The objective is to use different model representations, for example, environmental vairiables, ambiental variables, interactions and appliance consumptions. These models may receive input from different sensors distributed in the environment or mathematical formulae. There may also be the case where a different intrepretation of the values of a primary model or group of models may derive new ones, i.e., electric costs and carbon emission from electric consumption. Therefore, the user is aware of his impact on his environment.

System Architecture as proposed is a multi-agent system which encompasses a set of agents accountable for the assessment of current sustainability indicators in the environment. These agents communicate with the environment in order to retrieve data about it and use intelligent models to assess it based on sustainability indicators. Indeed, the proposed architecture uses 4 (four) different types of agents, namely:

- Sensing - an agent connected to sensors that is responsible to gather data about the environment, such as user presence or energetic consumption;

- Modeling - an agent responsible to model a representation of the essential aspects of a system which presents knowledge and relies on both mathematical and physical formulas as well as data from the sensing agents;

- Reasoning - an agent that reasons about perspectives, setting a method that fulfills the formal requirements for a theory of context, and offers an explanatory account of contextual reasoning in terms of information flow; 
- Actuator - an agent that acts on the environment and monitors the execution of those acts to reassure a correct behaviour on the system.

With this setup the environment has all the necessary agents to gather data, transform and process it into agent's knowledge models.

Sustainability Indicators available in Table 1 are in the form of the three key sustainability categories: economical metrics that include the running costs associated to the environment continuance versus the available income in the same period; social metrics that relate the presence and the possible interaction among the users that populated the environment; and environmental that assesses the the amount of carbon emissions that are generated. The values from the proposed indicator are interpreted as unsustainable if the value is below 1 or sustainable otherwise.

Table 1 Sustainability indicators

\begin{tabular}{ccc}
\hline Economical & Environmental & Social \\
\hline$\frac{\text { EnergeticCosts }}{\text { IncomeAvailable }}$ & $\frac{\text { EmissionTreated }}{\text { TotalEmission }}$ & $\frac{\text { TimeInside }}{\text { TimeOutside }}$
\end{tabular}

The overall sustainability of the environment is calculated by 1 , which denotes a compromise among the three main categories of sustainability indicators.

$$
S_{\text {index }}=\alpha \times I_{\text {economic }}+\beta \times I_{\text {environmental }}+\gamma \times I_{\text {social }}
$$

The equation presented in 1 has three qualifiers, one for each sustainability indicator. The values of such indicators are between 0 and 1 where their addition totals 1. All indicators are calculated either locally or for the entire setting which sums the assessment of all premisses. This way, if the environment is considered sustainable, the user may still assess changes in premises with low supportable standards.

\section{Tests and Results}

In order to set up initial scenarios both a simulation and a real environment were studied. On both environments it is possible to record consumptions, presence and emissions. User notification is made through a dedicated interface that displays environment configuration, its sensors, and the levels of consumptions and emissions.

For the simulated environment, a model of a traditional home with a bedroom, a living room, a bathroom, a kitchen and a hall was conceived. The actions simulated included user movement and the swapping of the state of the appliances (on or off). Electrical consumption, user presence and carbon emissions were modelled from simulated sensor values for a period of 3 days and a total of 201 actions simulated representing a user presence of about $58 \%$ in the environment each day. 
The Intelligent Systems Laboratory (ISLAB) at University of Minho was selected as the real environment. ISLAB is made of one room available to researchers where they can gather and work together. This room was modeled containing a set of appliances such as computers and lights. In this room it was also installed sensors which recorded user presence, luminance and temperature for the period of 5 days where 2 of those were holidays. The sensing and modeling agents were used to obtain model representation of user occupancy, lightning and temperature on the ISLAB.

Table 2 Results from simulation

\begin{tabular}{|c|c|c|c|}
\hline \multicolumn{4}{|c|}{ ISLAB Environment } \\
\hline Room & Carbon emission $(\mathrm{g})$ & Energetic Consumption (kW) & Presence $(\%)$ \\
\hline ISLAB & 7078.46 & 21.80 & 11 \\
\hline \multicolumn{4}{|c|}{ Home Environment } \\
\hline Room & Carbon emission $(\mathrm{g})$ & Energetic Consumption (kW) & Presence $(\%)$ \\
\hline Bedroom & 5.51 & 0.02 & 55 \\
\hline Kitchen & 3537.32 & 9.95 & 9 \\
\hline Living Room & 994.43 & 2.80 & 32 \\
\hline Bathroom & 3.47 & 0.01 & 1 \\
\hline Hall & 13.87 & 0.04 & 3 \\
\hline
\end{tabular}

Results from both simulations are presented in Table 2 according to the formulas presented in Table 1 and data simulated and obtained from sensors. In order to estimate an economic indicator, the user is supposed to have 1,5 euros per day for daily energy consumption. From these results it is possible to calculate the sustainable indexes defined in 1 which are 3.19, 0.67 and 1.38, in the home setup, and $1.92,0.67$ and 0.14 , in the ISLAB setup, for the economic, environmental and social indexes. It is also demonstrated, in the home environment, that it is economically viable, however, there is a penalization on the environmental indicator. This points out that the environment under study does not treat effciently emissions derived from its services. It is a potential source of improvement.

On the ISLAB results it is possible to demonstrate that if there is a budget of 1 euro for electricity on a daily basis then, sustainability indicators show that there is a penality not only in the environmental indicator but also in the presence indicator due to the limited time the room is occupied and its eletrical consumption. Changing the environment generates different simulation results, so simulation can be used to assess the impact of desired changes in the environment in terms of sustainability.

\section{Conclusion}

The proposed work features the perception of a multi-agent system able to handle sustainable monitoring and assessment in an intelligent environment. Furthermore, the use of formal models, was found to be a bright way to test and assess its sustainable actions and behaviours. It was found that it is possible to derive conclusions 
from the use of such systems in order to improve its efficiency and sustainability indicators. This is achieved with user awareness about his current situation, indicating which of his actions are more sustainable. As future work there is the need to further validate the model using real sensors in real world settings and design new metrics for sustainability indicators. The multi-agent system needs to be extended with the ability to monitor different environments in parallel, and explore connections and influences among them, such as a home and an office in the same environment. A recommender system may help in the improvement of the sustainability indicators.

Acknowledgements The research presented is partially supported by a portuguese doctoral grant, SFRH/BD/78713/2011, issued by the Fundaçäo da Ciência e Tecnologia (FCT) in Portugal.

\section{References}

1. Adomavicius, G., Tuzhilin, A.: Context-aware recommender systems. In: F. Ricci, L. Rokach, B. Shapira, P.B. Kantor (eds.) Recommender Systems Handbook, pp. 217-253. Springer US (2011)

2. Al-Waer, H., Clements-Croome, D.J.: Key performance indicators (kpis) and priority setting in using the multi-attribute approach for assessing sustainable intelligent buildings. Building and Environment 45(4), 799-807 (2009). URL http://centaur.reading.ac.uk/11750/

3. Aztiria, A., Izaguirre, A., Augusto, J.C.: Learning patterns in ambient intelligence environments: a survey. Artif. Intell. Rev. 34, 35-51 (2010). DOI 10.1007/s10462-010-9160-3

4. Chetty, M., Tran, D., Grinter, R.E.: Getting to green: understanding resource consumption in the home. In: Proceedings of the 10th international conference on Ubiquitous computing, UbiComp '08, pp. 242-251. ACM, New York, NY, USA (2008). DOI 10.1145/1409635.1409668

5. Ducatel, K., Bogdanowicz, M., Scapolo, F., Leijten, J., Burgelman, J.C.: Scenarios for Ambient Intelligence in 2010. Tech. rep., IST Advisory Group (2001). URL $\mathrm{ftp}: / / \mathrm{ftp}$. cordis.lu/pub/ist/docs/istagscenarios2010.pdf

6. Hagras, H., Doctor, F., Callaghan, V., Lopez, A.: An incremental adaptive life long learning approach for type-2 fuzzy embedded agents in ambient intelligent environments. IEEE Transactions on Fuzzy Systems 15(1), 41-55 (2007)

7. Lyon, A., Dahl: Achievements and gaps in indicators for sustainability. Ecological Indicators 17(0), 14 - 19 (2012). DOI 10.1016/j.ecolind.2011.04.032. Indicators of environmental sustainability: From concept to applications

8. Neves, J., Ribeiro, J., Pereira, P., Alves, V., Machado, J., Abelha, A., Novais, P., Analide, C., Santos, M., Fernndez-Delgado, M.: Evolutionary intelligence in asphalt pavement modeling and quality-of-information. Progress in Artificial Intelligence 1, 119-135 (2012). DOI 10.1007/s13748-011-0003-5. 10.1007/s13748-011-0003-5

9. Rui, C., Yi-bin, H., Zhang-qin, H., Jian, H.: Modeling the ambient intelligence application system: Concept, software, data, and network. Systems, Man, and Cybernetics, Part C: Applications and Reviews, IEEE Transactions on 39(3), 299 -314 (2009). DOI 10.1109/TSMCC.2009.2014390

10. Schilit, B., Adams, N., Want, R.: Context-aware computing applications. In: Mobile Computing Systems and Applications, 1994. WMCSA 1994. First Workshop on, pp. 85 -90 (1994). DOI 10.1109/WMCSA.1994.16

11. Singh, R., Murty, H., Gupta, S., Dikshit, A.: An overview of sustainability assessment methodologies. Ecological Indicators 9(2), 189-212 (2009)

12. Wang, K.I.K., Abdulla, W.H., Salcic, Z.A.: Ambient intelligence platform using multi-agent system and mobile ubiquitous hardware. Pervasive and Mobile Computing pp. 558-573 (2009) 